Pacific Journal of Mathematics

ON THE ZEROS OF A LINEAR COMBINATION O 


\section{ON THE ZEROS OF A LINEAR COMBINATION OF POLYNOMIALS}

\section{ROBERT VERMES}

In this paper we consider the location of the zeros of a complex polynomial $f(z)$ expressed as $f(z)=\sum_{k=0}^{n} a_{k} p_{k}(z)$ where $\left\{p_{k}(z)\right\}$ is a given sequence of polynomials of degree $k$ whose zeros lie in a prescribed region $E$. The principal theorem states that the zeros of $f(z)$ are in the interior of a Jordan curve $S=\{z ;|F(z)|=\operatorname{Max}(1, R)\}$ where $F$ maps the complement of $E$ onto $|z|>1$ and $R$ is the positive root of the equation $\sum_{k=0}^{n-1} \lambda_{k}\left|a_{k}\right| t^{k}-\lambda n\left|a_{n}\right| t^{n}=0$, with $\lambda_{k}>0$ depending on $E$ only. Several applications of this theorem are given. For example; if $\left\{p_{k}(z)\right\}$ is a sequence of orthogonal polynomials on $a \leqq z \leqq b$, then we give an ellipse containing all the zeros of $\sum_{k=0}^{n} a_{k} p_{k}(z)$.

Previous results. An extensive mathematical literature deals with the location of the zeros in the complex plane of a polynomial

$$
f(z)=a_{0}+a_{1} z+\cdots+a_{n} z^{n}
$$

with complex coefficients $a_{j}$. Cauchy derived practical bounds for the moduli of the zeros of (1) using the moduli of the coefficients $a_{j}$. In many investigations the polynomial (1) is not expressed as a linear combination of the sequence $\left\{z^{k}\right\}$, but as

$$
f(z)=b_{0}+b_{1} p_{1}(z)+\cdots+b_{n} p_{n}(z)
$$

where $\left\{p_{l k}(z)\right\}$ is a given sequence of polynomials. Cauchy's well known result (Marden [2], Th. 27, 1) was generalized by Turán [4] in the case where the expansion in (2) is the Hermite-expansion $e^{z^{2}} \sum_{k=0}^{n} b_{k}\left(e^{-z^{2}}\right)^{(k)}$. He obtained upper bounds for the moduli of the imaginary parts of the zeros, i.e., a "strip" where all the zeros of (2) are located. Specht [3], making use of the Christoffel-Darboux formula, extended these results to other sequences of orthogonal polynomials. In our Theorem 1 , we replace the "strip" with a bounded region, which will yield an ellipse in the case where the $\left\{p_{k}(z)\right\}$ is a sequence of orthogonal polynomials on a finite interval.

2. Cauchy type estimate. In the sequal we shall use the following notations: Let $E$ be a compact (infinite) set in the complex $z$-plane, whose complement $G$ is simply connected, $w=F(z)$ the univalent function which is defined on $G$ and maps $G$ conformally on $D:|w|>1$ such that the point at infinity in the two planes correspond to each 
other and also preserves direction there. The function $F(z)$ has the expansion in the point at infinity

$$
w=F(z)=\frac{1}{\tau} z+c_{0}+c_{1} z^{-1}+c_{2} z^{-2}+\cdots
$$

where $\tau$ is the transfinite diameter of $E$. If the boundary $B$ of $G$ is a Jordan curve, then according to a well known theorem (see Carathéodory [1]) the function $F(z)$ is continuous in the closure of $G$ and maps the boundary $B$ one-to-one onto $|w|=1$. We shall denote by $C_{R}$ the inverse image of the circle $|F(z)|=R(R>1)$.

With these notations we are able to state the following:

THeOREM 1. Let $G$ be the complement of a finite domain $E$ whose boundary $B$ is a Jordan curve. If $\mathscr{P}=\left\{p_{r}(z) ; E\right\}$ is a sequence of polynomials of exact degree $r$ whose zeros are in $E$, then the polynomial

$$
f(z)=a_{0} p_{0}+a_{1} p_{1}(z)+\cdots+a_{n} p_{n}(z)
$$

has its zeros in the closed interior of the Jordan curve $S=\{z ;|F(z)|=$ $\operatorname{Max}(1, R)\}$, where $R$ is the (only) positive root of the equation

$$
\lambda_{0}\left|a_{0}\right|+\lambda_{1}\left|a_{1}\right| t+\cdots+\lambda_{n-1}\left|a_{n-1}\right| t^{n-1}-\lambda_{n}\left|a_{n}\right| t^{n}=0 .
$$

The $\lambda_{r}$ are positive and depend only on $\mathscr{P}$ and $E$.

Proof. The rational function $\left(p_{r}(z) / p_{r+1}(z)\right)$ has the expansion in the neighborhood of the point at infinity:

$$
\frac{p_{r}(z)}{p_{r+1}(z)}=b_{1} z^{-1}+b_{2} z^{-2}+\cdots \quad\left(b_{1} \neq 0\right) .
$$

Define

$$
g_{r}(z)=\frac{p_{r}(z)}{p_{r+1}(z)} F(z) .
$$

Using (6) and (3) we obtain the following expansion for $g_{r}$ at $z=\infty$ :

$$
g_{r}(z)=d_{0}+d_{1} z^{-1}+d_{2} z^{-2}+\cdots \quad\left(d_{0} \neq 0\right) .
$$

Hence $g_{r}(z)$ is analytic in the domain $G$ and continuous in $G \cup B$. With the aid of the maximum modulus theorem we obtain:

$$
\left|g_{r}(z)\right| \leqq \operatorname{Max}_{z \in B}\left|\frac{p_{r}(z)}{p_{r+1}(z)}\right||F(z)|=\operatorname{Max}_{z \in B}\left|\frac{p_{r}(z)}{p_{r+1}(z)}\right|=m_{r},
$$


since $|F(z)|=1$ or $B$. From (7) and (8) we obtain the estimate

$$
\left|\frac{p_{r}(z)}{p_{r+1}(z)}\right| \leqq \frac{m_{r}}{|F(z)|} \quad \text { for } \quad z \in G
$$

For $r<n$

$$
\left|\frac{p_{r}(z)}{p_{n}(z)}\right|=\left|\frac{p_{r}(z)}{p_{r+1}(z)}\right|\left|\frac{p_{r+1}(z)}{p_{r+2}(z)}\right| \cdots\left|\frac{p_{n-1}(z)}{p_{n}(z)}\right| \leqq \frac{m_{r} m_{r+1} \cdots m_{n-1}}{|F(z)|^{n-r}} .
$$

Denote $\lambda_{r}=m_{r} m_{r+1} \cdots m_{n-1}$, then for $z \in G$

$$
\left|\frac{p_{r}(z)}{p_{n}(z)}\right| \leqq \frac{\lambda_{r}}{|F(z)|^{n-r}}
$$

Now, let $\zeta \in G$ be a zero of the polynomial in (4), then

$$
\left|a_{n}\right|\left|p_{n}(\zeta)\right| \leqq\left|a_{0}\right|+\left|a_{1}\right|\left|p_{1}(\zeta)\right|+\cdots+\left|a_{n-1}\right|\left|p_{n-1}(\zeta)\right|
$$

from which, after dividing by $p_{n}(\zeta) \neq 0$ and using (11), we obtain

$$
\left|a_{n}\right||F(\zeta)|^{n} \leqq \lambda_{0}\left|a_{0}\right|+\lambda_{1}\left|a_{1}\right||F(\zeta)|+\cdots+\lambda_{n-1}\left|a_{n-1}\right||F(\zeta)|^{n-1} .
$$

But this inequality implies that $|F(\zeta)| \neq R$, for $R$ is the root of (5). From the definition of $C_{R}$ it follows that $\zeta$ is in the closed interior of $C_{R}$. If $\zeta \in E$ then clearly $\zeta$ is in the interior of $C_{1}$, hence all the zeros of (4) lie in the closed interior of the Jordan curve

$$
S=\{z:|F(z)|=\operatorname{Max}(1, R)\},
$$

which proves the theorem.

As an application of this theorem, consider a sequence of polynomials $p_{r}(z)$ with leading coefficient one and whose zeros (which we assume lie in the interval $[-1,+1])$ separate each other. More precisely: if $z_{1, r}, z_{2, r}, \cdots, z_{r, r}$ are the zeros of $p_{r}(z)$ and $z_{0, r}=-1, z_{r+1, r}=$ 1 , then each interval $\left(z_{k, r}, z_{k+1, r}\right), k=0,1, \cdots, r$, contains exactly one zero of $p_{r+1}(z)$. The mapping function which maps the exterior of $[-1,+1]$ onto the exterior of the unit circle is given by

$$
w=F(z)=z+\left(z^{2}-1\right)^{1 / 2}
$$

where we take that branch of $z+\left(z^{2}-1\right)^{1 / 2}$ which becomes infinite at $z=\infty$. The locus $C_{R}=\{z ;|w|=R\}$ will be an ellipse with foci at $+1,-1$ and with semi axes $(1 / 2)\left(R+R^{-1}\right),(1 / 2)\left(R-R^{-1}\right)$. Now, if $R=2+3^{1 / 2}$ then $C_{R}$ is the ellipse with major axis 4 and minor axis $2 \cdot 3^{1 / 2}$. The distance of any point $u$ outside or on $C_{R}$ from the zeros of $p_{r}(z)(r=1,2, \cdots)$ is greater than 1 . Let $u$ be such a point; then 


$$
\left|\frac{p_{r}(u)}{p_{r+1}(u)}\right|=\left|\frac{\left(u-z_{1, r}\right)\left(u-z_{2, r}\right) \cdots\left(u-z_{r, r}\right)}{\left(u-z_{1, r+1}\right) \cdots\left(u-z_{r+1, r+1}\right)}\right| \leqq 1 ;
$$

for suppose that the minimum distance of $u$ from $z_{k, r+1}$ is attained at $z_{k_{0}, r+1}$, i.e.,

$$
\left|u-z_{k_{0}, r+1}\right|=\operatorname{Min}_{1 \leqq k \leqq r+1}\left|u-z_{k, r+1}\right|>1 .
$$

If we replace $z_{k, r}$ by $z_{k+1, r+1}$ when $k \geqq k_{0}$ and $z_{k, r}$ by $z_{k, r+1}$ when $k<k_{0}$ in (15), then the numerator is increased because the zeros are separated. Using (16) we obtain

$$
\left|\frac{p_{r}(u)}{p_{r+1}(u)}\right| \leqq \frac{1}{\left|u-z_{k_{0}, r+1}\right|} \leqq 1 .
$$

If the interior of $C_{2+3^{1 / 2}}$ is our domain $E$ in Theorem 1 , then it follows from (17) that all the $\lambda_{r}$ satisfy $0<\lambda_{r}<1 r=0,1,2, \cdots$ and we obtain the following:

THEOREM 2. Let $\left\{p_{r}(z)\right\}$ be a sequence of polynomials with leading coefficient 1. If all the zeros of $p_{r}(z)(r=0,1,2, \cdots)$ lie in $[-1,+1]$ and the zeros of $p_{r}(z)$ and $p_{r+1}(z)$ separate each other, then all the zeros of the polynomial

$$
f(z)=\sum_{r=0}^{n} a_{r} p_{r}(z)
$$

are in the ellipse

$$
\frac{x^{2}}{\left(R+R^{-1}\right)^{2}}+\frac{y^{2}}{\left(R-R^{-1}\right)^{2}}=1 / 4 \quad(z=x+i y)
$$

where $R=\max \left(2+3^{1 / 2}, \rho\right)$ and $\rho$ is the (only) positive root of

$$
\left|a_{0}\right|+\left|a_{1}\right| t+\left|a_{2}\right| t^{2}+\cdots+\left|a_{n-1}\right| t^{n-1}-\left|a_{n}\right| t^{n}=0 .
$$

In particular, if the sequence $\left\{p_{r}(z)\right\}$ in Theorem 2 is a sequence of orthogonal polynomials then the zeros of $p_{r}(z)$ and $p_{r+1}(z)$ separate each other and we have, for example, the following:

COROLLARY. If $f(z)=\sum_{r=0}^{n} a_{r} p_{r}(z)$ is a polynomial expounded in Legendre polynomials $p_{r}(z)$, then all the zeros of $f(z)$ are in the ellipse as given in Theorem 2.

We will use Theorem 1 to prove a result, Theorem 3 , which is analogous to Pellet's theorem (Marden [2], Th. 28, 1). Keeping the notation of Theorem 1, define: 


$$
d_{r}^{(k)}=\operatorname{Max}_{z \in B}\left|\frac{p_{r}(z)}{p_{k}(z)}\right| \quad(r=0,1,2, \cdots)
$$

then, as in (9)

$$
\left|\frac{p_{r}(z)}{p_{k}(z)}\right| \leqq \frac{d_{r}^{(k)}}{|F(z)|^{k-r}} \quad \text { for } \quad z \in G
$$

With the aid of inequality (21) we prove:

ThEOREM 3. Let E, G, B and $\left\{p_{r}(z)\right\}$ be as in Theorem 1. If for a polynomial

$$
f(z)=\sum_{r=0}^{n} a_{r} p_{r}(z) . \quad a_{k} \neq 0 \text { for some } k, \quad 0<k \leqq n
$$

the equation:

$$
H(t)=\sum_{r=0}^{k-1} d_{r}^{(k)}\left|a_{r}\right| t^{r}-d_{k}^{(k)}\left|a_{k}\right| t^{k}+\sum_{r=k+1}^{n} d_{r}^{(k)}\left|a_{r}\right| t^{r}=0
$$

has two positive roots $\rho$ and $R^{1} 1<\rho<R$, and if the only positive root of

$$
\sum_{r=0}^{k=1} d_{r}^{(k)}\left|a_{r}\right| t^{r}-d_{k}^{(k)}\left|a_{k}\right| t^{k}=0
$$

is greater or equal to 1 , then $f(z)$ has exactly $k$ zeros in or on $C_{\rho}$ and no zeros in the open ring Ext. $C_{\rho} \cap$ Int. $C_{R}$.

Proof. The region Ext. $C_{\rho} \cap$ Int. $C_{R}$, by assumption $(\rho>1)$ is contained in $G$, hence we will show that if $\zeta \in G$ is a zero of (22) then $\zeta \notin$ Ext. $C_{\rho} \cap$ Int. $C_{R}$. Because $p_{r}(\zeta) \neq 0$ and $\sum_{r=0}^{n} a_{r} p_{r}(\zeta)=0$ we have:

$$
\left|a_{k}\right| \leqq \sum_{\substack{r=0 \\ r \neq k}}^{n}\left|a_{r}\right|\left|\frac{p_{r}(\zeta)}{p_{k}(\zeta)}\right|
$$

Using inequality (21) we obtain

$$
\left|a_{k}\right| \leqq \sum_{\substack{r=0 \\ r \neq k}}^{n}\left|a_{r}\right| \frac{d_{r}^{(k)}}{|F(\zeta)|^{k-r}}
$$

and hence

$$
\begin{aligned}
& H(|F(\zeta)|)=\sum_{r=0}^{k-1} d_{r}^{(k)}\left|a_{r}\right||F(\zeta)|^{r} \\
& \quad-d_{k}^{(k)}\left|a_{k}\right||F(\zeta)|^{k}+\sum_{r=k+1}^{n} d_{r}^{(k)}\left|a_{r}\right|\left|F(\zeta)^{r}\right| \geqq 0 .
\end{aligned}
$$

1 If not all the $a_{r}(r<k)$ are zero, then the equation (23), according to the Descartes rule of sign, has two positive roots or has no positive roots at all. 
This last inequality tells us that $t=|F(\zeta)|<\rho$ or $t=|F(\zeta)|>R$, because the function $H(t)$ is negative only for $\rho<t<R$. We have shown that $\zeta \notin C_{t}$ when $\rho<t<R$ i.e., $\zeta \notin$ Ext. $C_{\rho} \cap$ Int. $C_{R}$.

To complete the proof of Theorem 3 we have to show that the closed interior of $C_{\rho}$ contains exactly $k$ zeros. We will do it by using a continuity argument. Define

$$
f(z ; s)=\sum_{r=0}^{k} a_{r} p_{r}(z)+\sum_{r=k+1}^{n} s a_{r} p_{r}(z) \quad(0 \leqq s \leqq 1)
$$

and the function

$$
H(t ; s)=\sum_{r=0}^{k-1} d_{r}^{(k)}\left|a_{r}\right| t^{r}-d_{k}^{(k)}\left|a_{k}\right| t^{k}+\sum_{r=k+1}^{n} d_{r}^{(k)} s\left|a_{r}\right| t^{r} .
$$

$H(t ; s)$, if $s=1$, has two positive roots $\rho(1)=\rho, R(1)=R$. If $s$ tends to 0 , the two roots $\rho(s)$ and $R(s)$ of (29) start to move; $\rho(s)$ decreases and $R(s)$ increases, and according to the first part of this theorem, Ext. $C_{\rho(s)} \cap$ Int. $C_{R(s)}$ is always zero free, hence the number of zeros $N(s)$ in the closed interior $C_{\rho(s)}(0 \leqq s \leqq 1)$ is a constant call it $N$. If $s \rightarrow 0$ then $R(s) \rightarrow \infty$ and $\rho(s) \rightarrow \rho^{*} \geqq 1$ by (24). But Theorem 1 applies to $f(z ; 0)$ and $H(t ; 0)$. Consequently the number of zeros in $C_{\rho}^{*}$ is exactly $k$, i.e., $N=k$ which completes the proof of Theorem 3.

In the preceding theorems we obtained bounds for all the zeros of (2) as function of all the coefficients $a_{r}$. However, if we restrict ourselves to $p+1$ fixed coefficients and $n-p$ arbitrary ones, are we able to find some bounds for $p$ zeros? In the case $f(z)=\sum_{r=0}^{n} a_{r} z^{r}$ Van Vleck [5] proved that essentially there is only one case in which bounds for $p$ zeros are derivable, i.e., if the fixed coefficients are the first $p$ consecutive ones and any other one from the remaining set. In other words, he showed that if one of the coefficients $a_{0}, a_{1}, \cdots, a_{p-1}$ is arbitrary, then at least $n-p+1$ zeros of $\sum_{r=0}^{n} a_{r} z^{r}$ may be made arbitrarily large in modulus. Perhaps it is interesting to note that this is the case in which the polynomial (2) is expressed with the aid of the sequence $p_{n}(z)$ in Theorem 1 . Suppose $a_{k}$ for some $k(0 \leqq k \leqq p-1)$ in $f(z)=\sum_{0}^{n} a_{r} p_{r}(z)$ is arbitrary. Let $\mathscr{L}>1$ be such that the distance of $C_{\mathscr{g}}$ from the origin is greater than a fixed large number $\delta$. Choose $a_{k}$ so large in modulus that the equation in (24) has a root greater than 1 and also

$$
d_{k}^{(k)}\left|a_{k}\right| \mathscr{L}^{k}<\sum_{\substack{r=0 \\ r \neq k}}^{n} d_{r}^{(k)}\left|a_{r}\right| \mathscr{L}^{r}
$$

But (30) implies that the equation $H(t)=0$ in (23) has a root $R>\mathscr{L}$. According to Theorem $3 n-k \geqq n-p+1$ zeros $z_{1}, z_{2}, \cdots, z_{n-k}$ of 
$f(z)$ are in Ext. $C_{R}$. Thus $\left|z_{j}\right|>\delta$ for $j=1,2, \cdots, n-k$, because Ext. $C_{\mathscr{R}} \subset$ Ext. $C_{\mathscr{O}}$.

\section{REFERENCES}

1. C. Carathéodory, Conformal representation, Cambridge, 1932.

2. M. Marden, The geometry of the zeros, Math. Surveys 3, Amer. Math. Soc., 1949. 3. W. Specht, Die Lage der Nullstellen eines Polynoms, Math. Nachrichten 16 (1956), 353-374.

4. P. Turàn, Hermite-expansion and strips for zeros of polynomials, Archive der Mathematik 5 (1954), 118-152.

5. E. B. Van Vleck, On limits to the absolute values of the roots of a polynomial, Bulletin de la Société Mathématique de France, 53 (1925), 105-125.

Received June 3, 1965. This research was supported in part by the N.S.F. Grant No. G-16135. The author wishes to express his graditude to Professor M. Marden for his constant encouragement.

McGill University, Montreal 



\section{PACIFIC JOURNAL OF MATHEMATICS}

\section{EDITORS}

H. SAMELSON

Stanford University

Stanford, California

J. P. JANS

University of Washington

Seattle, Washington 98105

\section{J. DuGUNDJI}

University of Southern California

Los Angeles, California 90007

RICHARD ARENS

University of California

Los Angeles, California 90024

\section{ASSOCIATE EDITORS}

E. F. BECKENBACH
B. H. NEUMANN

F. WOLF

K. YoSIDA

\section{SUPPORTING INSTITUTIONS}

\author{
UNIVERSITY OF BRITISH COLUMBIA \\ CALIFORNIA INSTITUTE OF TECHNOLOGY \\ UNIVERSITY OF CALIFORNIA \\ MONTANA STATE UNIVERSITY \\ UNIVERSITY OF NEVADA \\ NEW MEXICO STATE UNIVERSITY \\ OREGON STATE UNIVERSITY \\ UNIVERSITY OF OREGON \\ OSAKA UNIVERSITY \\ UNIVERSITY OF SOUTHERN CALIFORNIA
}

\author{
STANFORD UNIVERSITY \\ UNIVERSITY OF TOKYO \\ UNIVERSITY OF UTAH \\ WASHINGTON STATE UNIVERSITY \\ UNIVERSITY OF WASHINGTON \\ AMERICAN MATHEMATICAL SOCIETY \\ CHEVRON RESEARCH CORPORATION \\ TRW SYSTEMS \\ NAVAL ORDNANCE TEST STATION
}

Mathematical papers intended for publication in the Pacific Journal of Mathematics should be typewritten (double spaced). The first paragraph or two must be capable of being used separately as a synopsis of the entire paper. It should not contain references to the bibliography. Manuscripts may be sent to any one of the four editors. All other communications to the editors should be addressed to the managing editor, Richard Arens at the University of California, Los Angeles, California 90024 .

50 reprints per author of each article are furnished free of charge; additional copies may be obtained at cost in multiples of 50 .

The Pacific Journal of Mathematics is published monthly. Effective with Volume 16 the price per volume (3 numbers) is $\$ 8.00$; single issues, $\$ 3.00$. Special price for current issues to individual faculty members of supporting institutions and to individual members of the American Mathematical Society: $\$ 4.00$ per volume; single issues $\$ 1.50$. Back numbers are available.

Subscriptions, orders for back numbers, and changes of address should be sent to Pacific Journal of Mathematics, 103 Highland Boulevard, Berkeley 8, California.

Printed at Kokusai Bunken Insatsusha (International Academic Printing Co., Ltd.), No. 6, 2-chome, Fujimi-cho, Chiyoda-ku, Tokyo, Japan.

\section{PUBLISHED BY PACIFIC JOURNAL OF MATHEMATICS, A NON-PROFIT CORPORATION}

The Supporting Institutions listed above contribute to the cost of publication of this Journal, but they are not owners or publishers and have no responsibility for its content or policies. 


\section{Pacific Journal of Mathematics

Vol. 19, No. $3 \quad$ July, 1966

S. J. Bernau, The spectral theorem for unbounded normal operators .......

Lu-san Chen, Asymptotic behavior of solutions of parabolic equations of

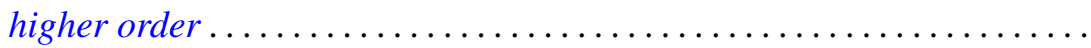

Lawrence William Conlon, An application of the Bott suspension map to the

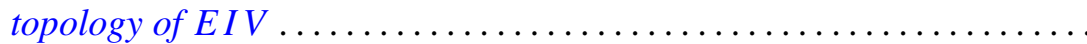

Neal Eugene Foland and John M. Marr, Sets with zero-dimensional kernels .........................................

Stanley Phillip Franklin and R. H. Sorgenfrey, Closed and image-closed

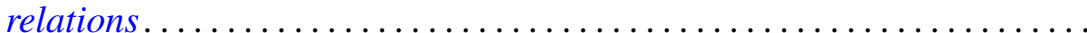

William Jesse Gray, A note on topological transformation groups with a

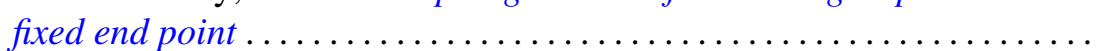

Myron Goldstein, $K$ - and L-kernels on an arbitrary Riemann surface ...... 449

George Joseph Kertz and Francis Regan, The exponential analogue of a generalized Weierstrass series .............................

Walter Leighton, On Liapunov functions with a single critical point ........ 467

Bernard Werner Levinger and Richard Steven Varga, On a problem of $O$.

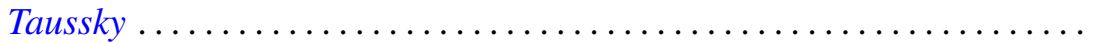

Lowell Duane Loveland, Tame subsets of spheres in $E^{3} \ldots \ldots \ldots \ldots \ldots . .489$

Erik Andrew Schreiner, Modular pairs in orthomodular lattices ......... 519

K. N. Srivastava, On dual series relations involving Laguerre polynomials ...............................

Arthur Steger, Diagonability of idempotent matrices.....

Walter Strauss, On continuity of functions with values in various Banach spaces...

Robert Vermes, On the zeros of a linear combination of polynomials ...

Elliot Carl Weinberg, On the scarcity of lattice-ordered matrix rings ....

Harold Widom, Toeplitz operators on $H_{p} \ldots \ldots \ldots \ldots \ldots$

Neal Zierler, On the lattice of closed subspaces of Hilbert space...

Irving Leonard Glicksberg, Correction to: "Maximal algebras and a theorem of Rado"

John Spurgeon Bradley, Correction to: "Adjoint quasi-differential operators of Euler type"

William Branham Jones, Erratum: "Duality and types of completeness in locally covex spaces".

Stanley P. Gudder, Erratum: "Uniqueness and existence properties of bounded observables" 